\title{
Behavioral Studies of Sound Localization in the Cat
}

\author{
Luis C. Populin and Tom C. T. Yin \\ Neuroscience Training Program and Department of Neurophysiology, University of Wisconsin-Madison, Madison, \\ Wisconsin 53706
}

\begin{abstract}
Using the magnetic search coil technique to measure eye and ear movements, we trained cats by operant conditioning to look in the direction of light and sound sources with their heads fixed. Cats were able to localize noise bursts, single clicks, or click trains presented from sources located on the horizontal and vertical meridians within their oculomotor range. Saccades to auditory targets were less accurate and more variable than saccades to visual targets at the same spatial positions. Localization accuracy of single clicks was diminished compared with the long-duration stimuli presented from the same sources. Control experiments with novel auditory targets, never associated with visual targets, demonstrated that the cats localized
\end{abstract}

the sound sources using acoustic cues and not from memory.

The role of spectral features imposed by the pinna for vertical sound localization was shown by the breakdown in localization of narrow-band (one-sixth of an octave) noise bursts presented from sources along the midsagittal plane. In addition, we show that cats experience summing localization, an illusion associated with the precedence effect. Pairs of clicks presented from speakers at $\left( \pm 18^{\circ}, 0^{\circ}\right)$ with interclick delays of $\pm 300 \mu \mathrm{sec}$ were perceived by the cat as originating from phantom sources extending from the midline to approximately $\pm 10^{\circ}$.

Key words: sound localization; behaving cat; animal psychophysics; head-fixed cat; summing localization; bandpass stimuli
The domestic cat has been widely used for studies of the neural circuits and mechanisms underlying sound localization. Anatomical studies have established the basic connectivity of the circuits, and single-cell recordings in anesthetized preparations have characterized the physiological properties of cells in most auditory nuclei (Phillips and Brugge, 1985; Irvine, 1986; Yin et al., 1997). Little is known, however, about the cat's ability to make absolute localization judgments. Behavioral studies have required cats to indicate where they perceive sound to originate by walking to the source (Casseday and Neff, 1973; Jenkins and Masterton, 1982; Jenkins and Merzenich, 1984) or to indicate whether the position of a test sound is different from the position of a reference sound in minimum audible angle paradigms using conditioned avoidance (Martin and Webster, 1987; Heffner and Heffner, 1988). These experiments do not directly address the cat's capabilities to localize the absolute position of a sound in space. Others have studied the head-orienting response, either with videotape (Thompson and Masterton, 1978; Beitel and Kaas, 1993) or electromagnetic sensor (May and Huang, 1996), which ignores eye position and assumes that the cat points its nose at the target. We believe it is more likely that the cat naturally looks at the target, and therefore we monitor eye position while keeping the head fixed.

To localize sound sources along the horizontal plane, interaural time and level differences for low- and high-frequency signals, respectively, provide the major cues (Stevens and Newman, 1936; Mills, 1958; Casseday and Neff, 1973). For localizing sources located along the midsagittal plane, for which interaural disparities are minimal if the ears are oriented symmetrically, features in

Received July 30, 1997; revised Dec. 15, 1997; accepted Dec 23, 1997.

This work was supported by National Institutes of Health Grants DC00116 and DC02840. We acknowledge the contributions of Ravi Kochhar and Jane Sekulski of the Neurophysiology Department and Richard Olson and Dan Yee of the Medical Electronics Laboratory of the University of Wisconsin Medical School. We are also grateful to Dr. Doris Kistler for assistance with statistical methods.

Correspondence should be addressed to Luis C. Populin, Department of Anatomy, University of Wisconsin, 1300 University Avenue, Madison, WI 53706.

Copyright (C) 1998 Society for Neuroscience $0270-6474 / 98 / 182147-14 \$ 05.00 / 0$ the spectrum of the acoustic signals reaching the eardrum seem important; these spectral notches and peaks are imposed by the filtering characteristics of the pinna and ear canal (Wiener et al., 1966; Gardner and Gardner, 1973; Hebrank and Wright, 1974a, 1974b; Butler, 1975; Phillips et al., 1982; Musicant et al., 1990; Middlebrooks, 1992; Rice et al., 1992; Wightman and Kistler, 1994). If these spectral cues are important for localization, then narrow band stimuli should be difficult to localize.

The mechanisms outlined above apply for the localization of single sound sources. To determine the position of a sound source in enclosed spaces in which there are echoes, the nervous system must deal with multiple copies of the original signal that result from reflections. The precedence effect (Wallach et al., 1949; Haas, 1951) is thought to facilitate localization in such reverberant environments (Hartmann, 1983). The effect can be demonstrated by presenting pairs of clicks with an interclick delay (ICD) from speakers located symmetrically about the subject. ICDs of $<1 \mathrm{msec}$ result in what has been termed summing localization, in which human subjects hear a single acoustic event originating from a phantom source localized toward the leading source (Blauert, 1983). Summing localization has been systematically studied in human subjects (Blauert, 1983; Perrott et al., 1987, 1989; Litovsky and Macmillan, 1994), who can verbally report the perceived location of the phantom source, but there are no comparable studies in cats. Both psychophysical (Cranford, 1982) and physiological (Yin, 1994) studies suggest that cats experience a similar effect, but direct demonstration is lacking.

The primary goal of the present study was to determine the accuracy with which cats localize the sources of acoustic stimuli of various types: long-duration, broad-band noise bursts; transients (single clicks); and narrow-band noise bursts. In addition, we sought to determine whether cats experience summing localization.

Preliminary results have been presented earlier (Populin and Yin, 1995, 1996). 


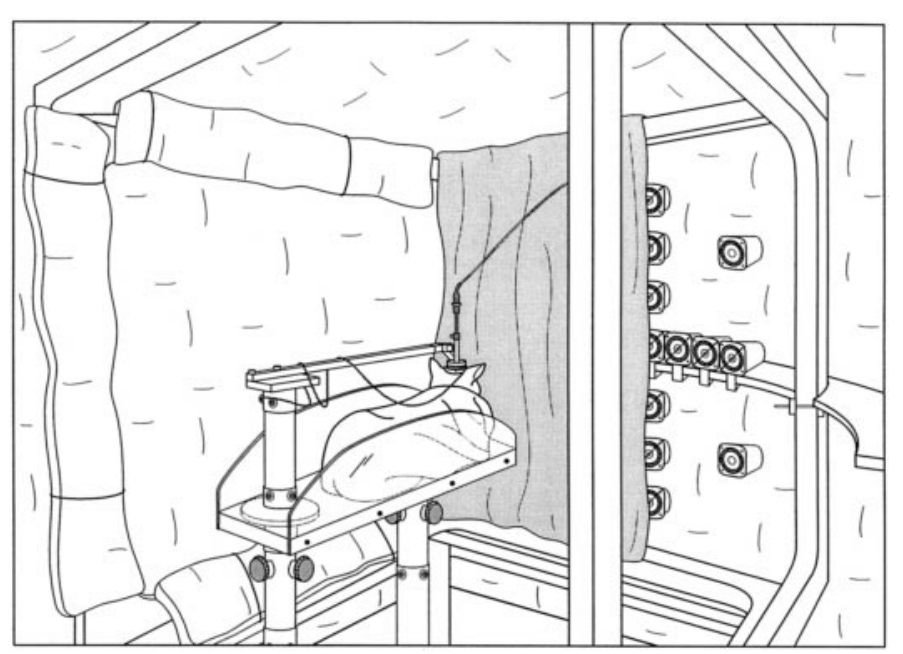

Figure 1. Drawing of the experimental setup. The interior of the recording chamber and the cubic frame housing the field coils of the magnetic search coil system are covered with foam to attenuate acoustic reflections; some panels have been removed for illustrative purposes. A cat is shown inside a canvas bag on a platform with its head fixed from behind by the holding bar. This animal was prepared for physiological recordings with a microdrive mounted on a recording chamber on top of the head. Multiple acoustic speakers are mounted in front of the cat behind a black cheesecloth curtain with LEDs positioned at the center of most of the speakers.

\section{MATERIALS AND METHODS}

Subjects and surgery. Four domestic cats, selected for their friendliness and clean middle ears, were used in these experiments. All surgical and experimental procedures used were in accordance with the National Institutes of Health guidelines. Before training, we implanted a small head post (modified from May et al., 1991) and fine wire coils (AS631, Cooner Wire Co., Chatsworth, CA) in each eye (Judge et al., 1980) and in each pinna (AS633, Cooner) under sterile surgical conditions. Anesthesia was induced with an intramuscular injection of ketamine and acepromazine (20 and $0.2 \mathrm{mg} / \mathrm{kg}$, respectively) and maintained during the remainder of the procedure with intravenous sodium pentobarbital or gas (halothane or isofluorane). Intravenous injections of atropine sulfate $(0.5 \mathrm{mg} / \mathrm{kg})$ were given to prevent obstruction of respiratory tracts with mucous buildup. A subcutaneous dose of the analgesic ketoprofen (2.0 $\mathrm{mg} / \mathrm{kg}$ ) was administered postoperatively daily as needed until recovery was complete.

Experimental setup: eye movement recording and stimulus presentation. All experiments were conducted in a sound-attenuated recording room $(2.2 \times 2.5 \times 2.5 \mathrm{~m})$. The inside of the room and the major pieces of equipment were covered with $10.2 \mathrm{~cm}$ Sonex foam (Ilbruck, Minneapolis, MN) to minimize acoustic reflections (Fig. 1). Eye movements were recorded with the magnetic search coil technique (Fuchs and Robinson, 1966). The analog output of the coil system (CNC Engineering, Seattle, WA), which has a resolution of $0.1^{\circ}$, was digitally sampled at $500 \mathrm{~Hz}$ with a 12 bit analog-to-digital converter.

Visual and auditory stimuli were presented from 15 light-emitting diode (LED)-speaker assemblies positioned within the cat's oculomotor range in an arc of $62 \mathrm{~cm}$ in radius. Each of the $15 \mathrm{LED}$-speaker units consisted of a Radio Shack super tweeter (modified to transduce low frequencies), outfitted with a $2.0-\mathrm{mm}$-diameter $\operatorname{red}\left(\lambda_{\max }=635 \mathrm{~nm}\right)$ LED in the center. Each LED subtended a visual angle of $0.2^{\circ}$. The 15 speakers were selected from a large set on the basis of matching frequency responses. The cat's view of the transducers was blocked by a dark cheesecloth screen (Fig. 1) that allowed sounds to be heard and LEDs to be seen when lit.

The waveforms used to generate the acoustic stimuli were played by a digital stimulus system (Rhode, 1976). These signals were fed through mercury relays that selected any of the 15 available channels and connected to 15 audio amplifiers. This arrangement, which allowed the background noise of the amplifiers to play continuously through the speakers, was found to be preferable to one in which a single audio amplifier drove all 15 speakers through the mercury relays, because in the latter case the cats were able to hear a faint transient when the back- ground noise of the amplifier was switched to a different speaker. Two other precautions were taken to minimize the possibility that switching transients, rather than controlled acoustic signals, could provide the cats with information as to which speaker was selected. First, the selection of the speaker, the process of setting the electronic connection to gate the output of the digital stimulus system to the appropriate amplifier and speaker, was performed shortly after the synchronization pulse (Fig. 2, $S Y$ ) that marked the onset of a trial, rather than just before the acoustic stimulus was turned on, to temporally dissociate the selection artifact from the acoustic signal. Second, we selected all speakers rapidly in succession followed by deselection of all but the speaker to be used, thereby producing transients in all channels.

To lessen the possibility that differences in speaker characteristics may assist in identifying and localizing individual speakers, we roved the level of each auditory trial over a $16 \mathrm{~dB}$ range in $2 \mathrm{~dB}$ steps, above the empirically determined $75 \%$ correct threshold. Evidence that the cats were using the acoustic stimuli, and not some artifact, to localize sounds came from the inability of the cats to perform the task when the sound pressure level of the stimuli was highly attenuated. Such was not the case before we implemented the above procedures.

Acoustic stimuli were broad-band $(0.1-25 \mathrm{kHz})$ noise and clicks $(100$ $\mu \mathrm{sec}$ duration). In the experiments using bandpass noise stimuli, we digitally filtered our standard wide-band noise to one-sixth of an octave wide (with $1000 \mathrm{~dB} /$ octave filter slopes) at center frequencies of 1, 2, 4, 8 , and $12 \mathrm{kHz}$. The entire experimental setup, including eye movement data acquisition, the digital stimulus system, and the speaker selection circuit, was controlled by a Microvax-2 computer (Digital Equipment Co., Maynard, MA).

The head of the cat was immobilized and held in the center of the cube housing the field coils of the magnetic search coil system with a horizontal bar machined to fit the head post from behind the cat's head (Fig. 1). This arrangement, adapted from McHaffie and Stein (1983), permitted immobilizing the cat's head and mounting a recording chamber while minimizing any disruption of vision, hearing, or pinna movements.

Eye coil calibration. The eye coil was calibrated with a behavioral procedure that relied on the natural instinct of the cat to look at a small light source that suddenly appears in the visual field. Adjustments were made to the polarity of the horizontal and vertical channels to match the actual direction of the cat's eye movements. The output of the coil system was recorded when the cat's eye assumed a stationary position at the end of eye movements evoked by visual stimuli presented from known positions. The vertical and horizontal components of eye position were fit separately with linear equations. The coefficients obtained from the fitting procedure were used by the data collection and analysis software to convert the voltage output of the coil system to degrees of visual angle.

Sound localization training and experimental tasks. The cats were fooddeprived during the day. A reward, made of finely ground cat chow mixed with canned cat food and water to the consistency of a paste, was delivered to the cat by air pressure regulated by solenoids after each successful trial. We monitored body weight daily to ensure that it remained within $15 \%$ of the original weight.

Initially the cats were shaped under manual control by rewarding them when they made saccades toward the correct location shortly after the presentation of LEDs. Then, under computer control we had the cats work on the various tasks shown in Figure 2 while, as performance improved, slowly decreasing the size of the electronic acceptance windows and increasing the duration of the fixation period and number of trials.

A fixation trial is shown in Figure $2 A$. The sequence of behavioral events is plotted at the bottom, and the square wave and the envelope of a noise burst represent visual and auditory stimuli, respectively. A schematic eye movement is shown at the top. The stimuli, which could be visual and acoustic combined or either modality by itself, were presented from any of the available target locations. The cat's task was to look to the stimuli and to maintain fixation within the acceptance window surrounding the target for the duration of the stimulus until a reward was delivered.

The saccade task also begins with fixation of LED 1, followed by the presentation of a target (visual, auditory, or both; Fig. $2 B$ ), the onset of which coincides with the offset of the fixation event. The cat was required to maintain fixation on LED 1 until it was turned off and then to move its eyes to the location of the target within a set time window $(1500 \mathrm{msec})$ and maintain fixation until it was turned off.

Most cats were able to perform saccadic eye movements to either visual or auditory targets in the first experimental sessions (e.g., Cat09), 


\section{A. Fixation}
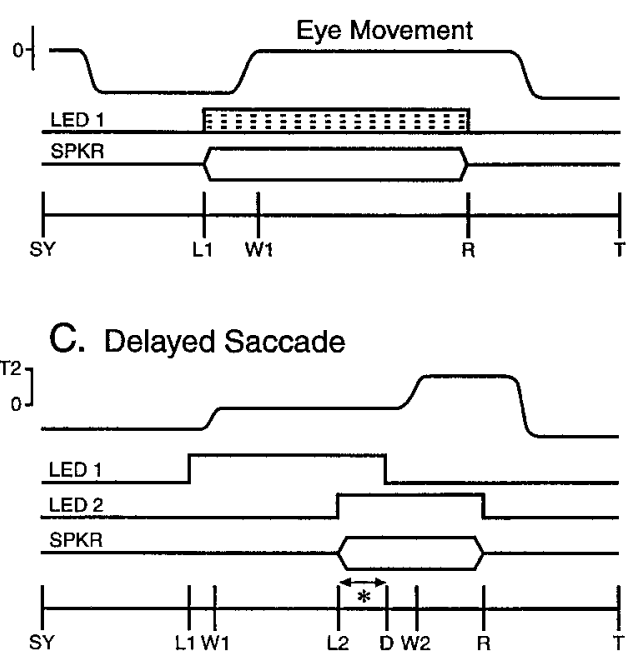

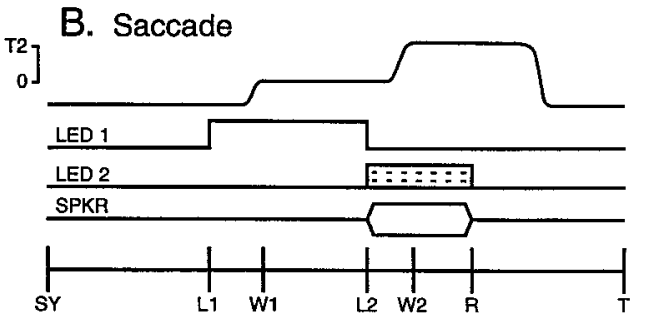

\section{Summing Localization}

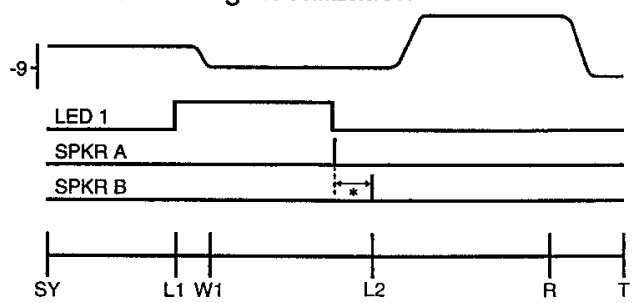

Figure 2. Schematic diagram of the behavioral tasks. $A$, Fixation; $B$, saccade; $C$, delayed saccade; and $D$, summing localization. Each panel shows the following from top to bottom: the expected eye movement, visual $(L E D)$ and auditory $(S P K R)$ target onset and offset, and the sequence of the behavioral events. Targets could be visual, auditory, or combined modality. SY, Synchronization pulse stored in the computer record to mark start of the trial; $L 1$, fixation LED onset; $W 1, W 2$, eye into acceptance window; $D$, delay; $L 2$, target onset; $R$, reward; $T$, end of trial. $A$, Fixation task. The animal was required to saccade to the target and to maintain fixation within the acceptance window surrounding the target. The broken lines within the LED represent the progressive dimming of the visual component of the combined stimuli used during the early stages of sound localization training. $B$, Saccade task. At the end of the fixation event $(L E D 1)$, another target was presented at a different location. To receive a reward the cat had to fixate LED 1 and then saccade to the location of the newly presented target until the reward $(R)$ was delivered. $C$, Delayed saccade. The target was presented before the offset of the fixation event $(L E D 1)$. The signal for the cat to move to the target was given by the offset of $L E D 1$; thus the delay, indicated by the asterisk, is the time during which $L E D 1$ and target (LED 2 or SPKR) overlap. D, Summing localization. Coinciding with the end of a fixation event (LED 1), a pair of $100 \mu$ sec clicks is presented from two speakers at $\left( \pm 18^{\circ}, 0^{\circ}\right)$ with ICDs (indicated by the asterisk) ranging over $\pm 1000 \mu$ sec.

but some were not able to orient to acoustic stimuli alone (e.g., Cat07). In these cases we presented both visual and auditory stimuli from the same location and then progressively dimmed the LED (Fig. $2 A, B$ dashed target traces) until it was extinguished.

To temporally dissociate the onset of the target and the signal to make a saccade, we used the delayed saccade task (Fig. $2 C$ ), in which the target (LED 2 or SPKR) was presented some time before the fixation LED 1 was extinguished. This results in a period during which the fixation LED and the target are both on (Fig. 2C, asterisk). The offset of LED 1 is the signal to make a saccade.

For all of the tasks shown in Figure 2, the duration of various events (e.g., W1-R, W1-L2, and L2-R intervals) were varied from one task type to another and within tasks, so that the cat could not predict how long any particular event might last. In addition, there was a temporal window (1500 msec after target appearance) within which the cat had to saccade into the acceptance window or the trial was judged a miss, in which case no reward was delivered, and a penalty in the form of a time-out period (double the intertrial time) was administered. The duration of the intertrial period varied from cat to cat $(4-8 \mathrm{sec})$ to allow the animal enough time to lick the reward from the feeding tube.

Summing localization: stimuli and experimental procedures. Summing localization was probed with stimuli consisting of a pair of $100 \mu$ sec clicks (Fig. $2 D)$, delivered from two speakers located at $\left( \pm 18^{\circ}, 0^{\circ}\right)$. The ICD within a click pair (Fig. $2 D$, asterisk) ranged between $\pm 1000 \mu$ sec. By convention, a positive ICD denotes the leading click of a pair to be to the right of the subject. Two different conditions were run: (1) we varied the ICD; and (2) we maintained the ICD at 0 but varied the level of one of the two clicks (left or right).

Reward contingencies. The cats were required to look at visual or auditory targets by maintaining eye position within a square electronic acceptance window centered on the target. Once the eye entered the window, the fixation time for the target was started, and the eye had to remain within the window or else the trial was terminated with a time-out. Because of differences in the accuracy with which visual and acoustic targets were localized, the windows were of different sizes; for visual targets the window was $2-5^{\circ}$, and for broad-band auditory targets the window was $6-12^{\circ}$ (see below).

The size of the acceptance window in any behavioral task, which was kept constant throughout an experimental session, was determined by balancing two conflicting considerations. To obtain optimal behavioral performance from the cat, the window should be small so that the cat could not relax and be rewarded for lackadaisical work. On the other hand, we wanted the cat to tell us by its eye movements where it heard the sound, not where it learned to get a reward. Ideally then, the window should be large enough to encompass all diligent attempts to look at the target but small enough to prevent half-hearted attempts at being rewarded. There is no way of knowing with our preparation whether inaccurate saccades were attributable to inattentiveness or to mislocalization. This is an inherent limitation of this paradigm. Adding to this difficulty is that the behavioral tasks differ in their difficulty, as judged by human observers, and that the cats' motivational level varied, as judged by how eagerly they approached the tasks of the day.

For most of our behavioral tasks the acceptance window was centered about the target, and a reward was given to the cat when it satisfied the spatial and temporal requirements. However, in some of the experiments in which the perceived location of the sound was an illusion (e.g., the summing localization) or was expected to be mislocalized (e.g., the narrow band signals), it was unclear where the correct target lies. In these cases, we wished to avoid training the cat to respond in a certain way by rewarding it only for particular responses. For these experiments we adopted a different strategy in which such trials were presented at low probability (5-10\%), randomly intermingled with other normal localization trials, and the cats were always rewarded, regardless of the response.

Finally, the acceptance window was only used during the experiment to determine whether to deliver a reward and did not affect the estimates of localization accuracy, because the analysis included final eye positions from both successful and unsuccessful trials. Trials in which the cat never entered the acceptance window for the initial LED, broke fixation of the LED before the target was turned on, or did not make an overt response (such as when the eyes never left the fixation window) were considered no responses and not included in the data analysis.

Data analyses. The dependent variable of interest was gaze position, which in the head-fixed animal is equivalent to eye position, after eye movements evoked by auditory or visual stimuli. To measure final eye position we used eye velocity to define the beginning and end of eye movements, as in Figure 3. The beginning and end of an eye movement were defined as the time at which fixation ended and restarted, respectively. The end of fixation was the time at which the amplitude of the eye velocity trace first exceeded 2 SDs of the mean baseline (Fig. 3, Fixation criterion). The mean baseline was computed from a portion of the velocity trace from $100 \mathrm{msec}$ before to $30 \mathrm{msec}$ after the onset of the stimulus, during which time the eye was expected to be stationary. Conversely, the return to fixation was defined as the time at which the amplitude of the velocity returned to within 2 SDs of the mean baseline. Identical steps were followed for the vertical component of eye position. In trials with corrective eye movements, such as the example shown in Figure 3, the return to fixation was measured at the end of the last corrective movement. Eye movements after the reward were not considered.

The presence of a slow tail in some of the eye movements (L. C. Populin and T. C. T. Yin, unpublished observations) could have led us to 
Figure 3. Criterion for determining the return to fixation. Horizontal eye position (thin trace) and velocity (heavier trace) from a visual trial to a target located at $\left(18^{\circ}, 0^{\circ}\right)$ are plotted as a function of time. The horizontal dotted line illustrates the fixation criterion. The vertical dotted line, drawn at the point at which the velocity trace intersects the fixation criterion line, illustrates the return to fixation. Final eye position is defined as the position at the time of return to fixation.

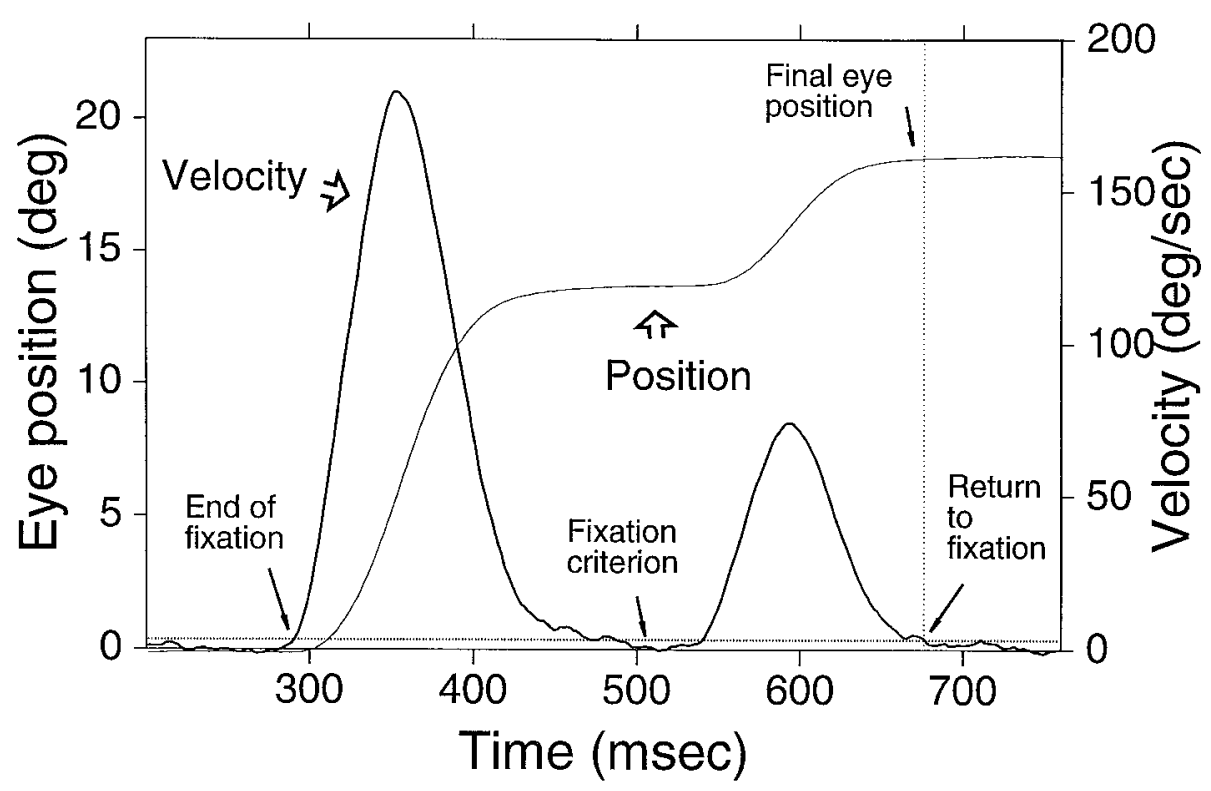

We quantified accuracy by localization errors, measured as the magnitude of the vector connecting each final eye position to its corresponding target (Fig. 6). All four cats were more accurate in looking to visual than auditory targets. In general, cats did not look to the same position for auditory and visual stimuli, despite the identical position of the transducers. There were only two cases in which the visual and auditory confidence intervals overlapped, at $\left(18^{\circ}, 0^{\circ}\right)$ in the data set of Cat05 and at $\left(0^{\circ}, 18^{\circ}\right)$ in the data set of Cat07 (Fig. 5), and thus were not significantly different.

In general, all cats underestimated the eccentricity of auditory targets. Unlike visual localization, in which most cats performed similarly, noticeable differences were observed in sound localization among all subjects. With one exception (see below), all four cats looked to distinct locations of space, indicating that they were able to localize sounds as originating from the four directions as well as to distinguish between the two different sound sources in each direction.

It was not uncommon for cats to develop biases, expressed as clear preferences for or against targets located in one or two quadrants. Occasionally these biases changed with time, and difficulties were seen with other targets. For example, Cat05 was much more accurate localizing the two auditory targets to its right than those to its left (Figs. 5, 6). Cat06, on the other hand, had difficulties with the target at $\left(18^{\circ}, 0^{\circ}\right)$ at the time these data were collected.

For targets along the vertical axis, the general trend was larger errors for targets located below the horizontal axis. Cat05 and Cat06 were able to localize targets along the vertical axis as originating from four distinct sources, although with errors. Cat07 and Cat09, however, were more accurate localizing the two targets above the horizontal plane. In the upward direction Cat07 overshot the target at $\left(0^{\circ}, 9^{\circ}\right)$, a rare finding for an auditory target, and its final eye positions for the visual and auditory targets at $\left(0^{\circ}, 18^{\circ}\right)$ overlapped. In the downward direction Cat 09 could not distinguish between the targets at $\left(0^{\circ},-14^{\circ}\right)$ and $\left(0^{\circ},-23^{\circ}\right)$, as indicated by the overlap between the confidence intervals in Figure 5 (stippled half-circle and horizontal ellipse), and Cat07 did not respond at all to the stimuli presented from the target at $\left(0^{\circ},-23^{\circ}\right)$.

Final eye position for visual targets was comparable in Cat05, location of auditory targets, although with less accuracy than to visual targets. 
Visual

\section{Auditory}

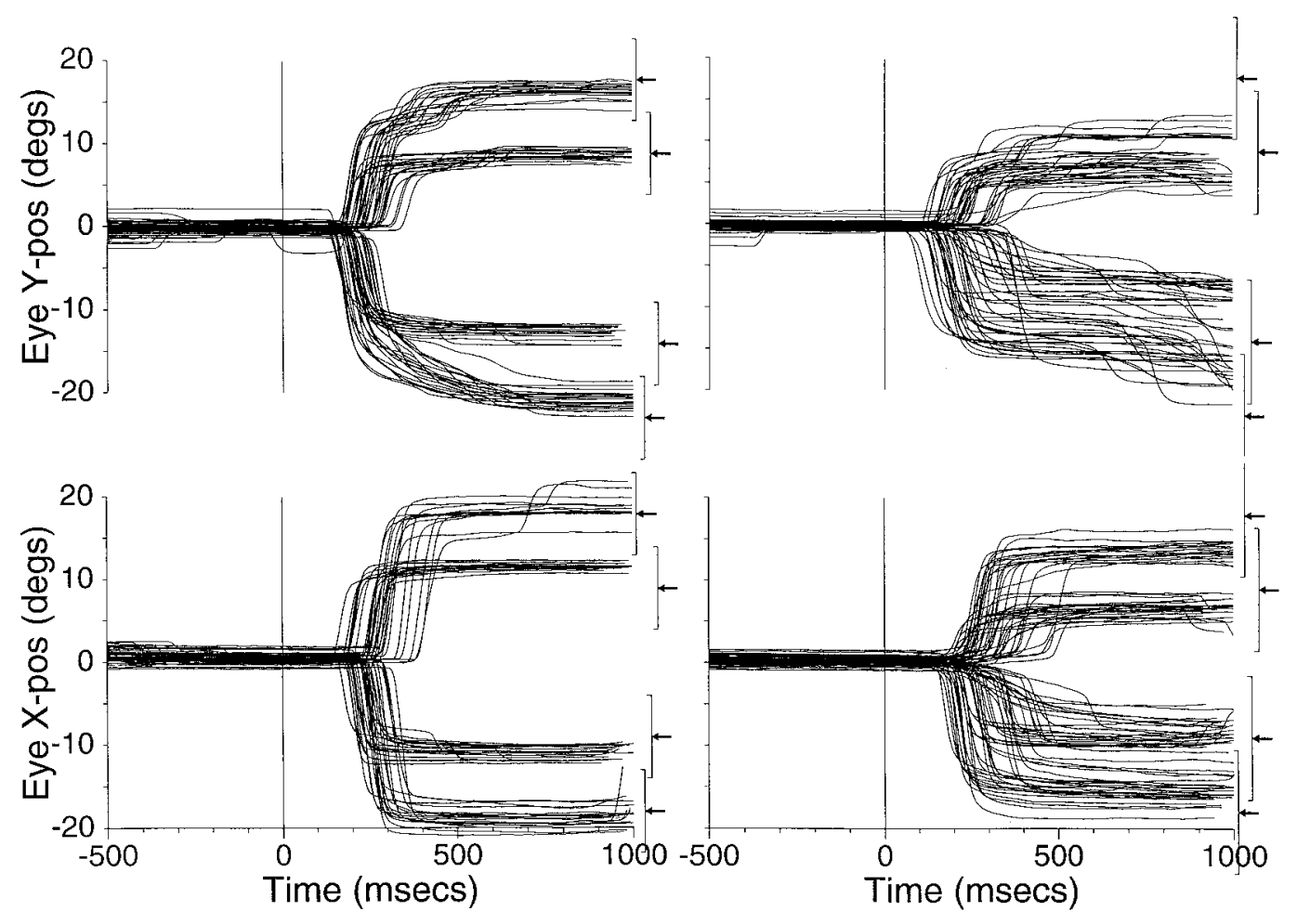

Figure 4. Typical eye movements of Cat06 to visual and auditory targets during the saccade task. The main component, either vertical or horizontal, of successful eye movements from the primary position $\left(0^{\circ}, 0^{\circ}\right)$ to targets located along the main vertical $\left(0^{\circ}, 18^{\circ} ; 0^{\circ}, 9^{\circ} ; 0^{\circ},-14^{\circ} ; 0^{\circ},-23^{\circ}\right)$ and horizontal $\left( \pm 18^{\circ}, 0^{\circ} ; \pm 9^{\circ}, 0^{\circ}\right)$ axes are plotted as a function of time and synchronized to stimulus onset (time, 0 msec); failed trials are omitted for clarity. The arrows to the right illustrate the position of the target for the component plotted, and the brackets illustrate the sizes of the acceptance window $\left( \pm 5^{\circ}\right.$ for visual, $\pm 7.5^{\circ}$ for auditory) surrounding each target. The number of trials included is given by $n=344$ (183 auditory and 161 visual).

Cat06, and Cat09. Cat07, on the other hand, was considerably less accurate than the other cats. On the horizontal domain it undershot the far peripheral targets on the left and right, and on the vertical domain it accurately pointed to targets located above the primary position but undershot those located below. Its poor performance was not attributable to the coils restricting its eye movements, because testing with a hand-held red laser pointer, which provided a strong stimulus for cats to track, indicated that this cat was able to move its eyes beyond $\pm 20^{\circ}$ (data not shown).

\section{Localization of transient stimuli: brief visual stimuli and single clicks}

For the previous series of experiments we tried to create favorable conditions for localizing sound within the constraints of our experimental setup. Accordingly, we chose long-duration, broadband noise to provide more exposure time and the opportunity for corrective saccades by leaving the stimulus on after the initial response was completed. But under natural conditions, cats must also be able to localize transient stimuli. Thus, as a comparison we tested two cats with a single $100 \mu \mathrm{sec}$ click and $25 \mathrm{msec}$ visual stimuli. Saccade trials of this type were introduced randomly among trials of other types within an experimental session.

The final eye position summary of these experiments is shown in Figure 7, and the corresponding absolute localization errors are shown in Figure 8. Visual localization was affected in the two animals, albeit in a different manner. Cat06's performance suffered an overall reduction, with localization of visual targets on the horizontal plane affected the most. Cat09's performance was most affected for visual targets located below the horizontal plane.

For the transient auditory stimuli both cats' performances were also affected. Cat06 was able to localize the targets above, left, and right of the primary position, and although it was able to point downward for the targets below, it could not distinguish between the two sources $9^{\circ}$ apart. Although Cat09 grossly undershot all horizontal targets, the final eye positions indicated that it perceived all four different sources in different locations. Its performance suffered the most for vertical localization; e.g., the mean final eye position for the target at $\left(0^{\circ},-14^{\circ}\right)$ is above the primary position. The increased difficulty of this task is reflected in the higher percentage of no responses: 45 compared with $8 \%$ in saccade trials to long-duration noise targets.

\section{Sound localization in the delayed-saccade paradigm}

To contrast with the previous conditions in which the cat had to localize and execute its responses from memory, we also studied the effect of longer exposure to the stimuli using the delayed saccade paradigm (Fig. $2 C$ ) in one cat. The delay was randomly varied between 300 and $700 \mathrm{msec}$ in $100 \mathrm{msec}$ steps; we show the $500 \mathrm{msec}$ delay in Figure 9. The horizontal component of successful eye movements to horizontal targets in the delayed saccade task is shown in Figure 9, $A$ and $B$, with all traces synchronized to the onset of the stimulus at $0 \mathrm{msec}$. Final eye positions, including successful and failed trials, are shown in Figure $9 C$, and the magnitude of the errors is shown in Figure $9 D$. The acoustic 


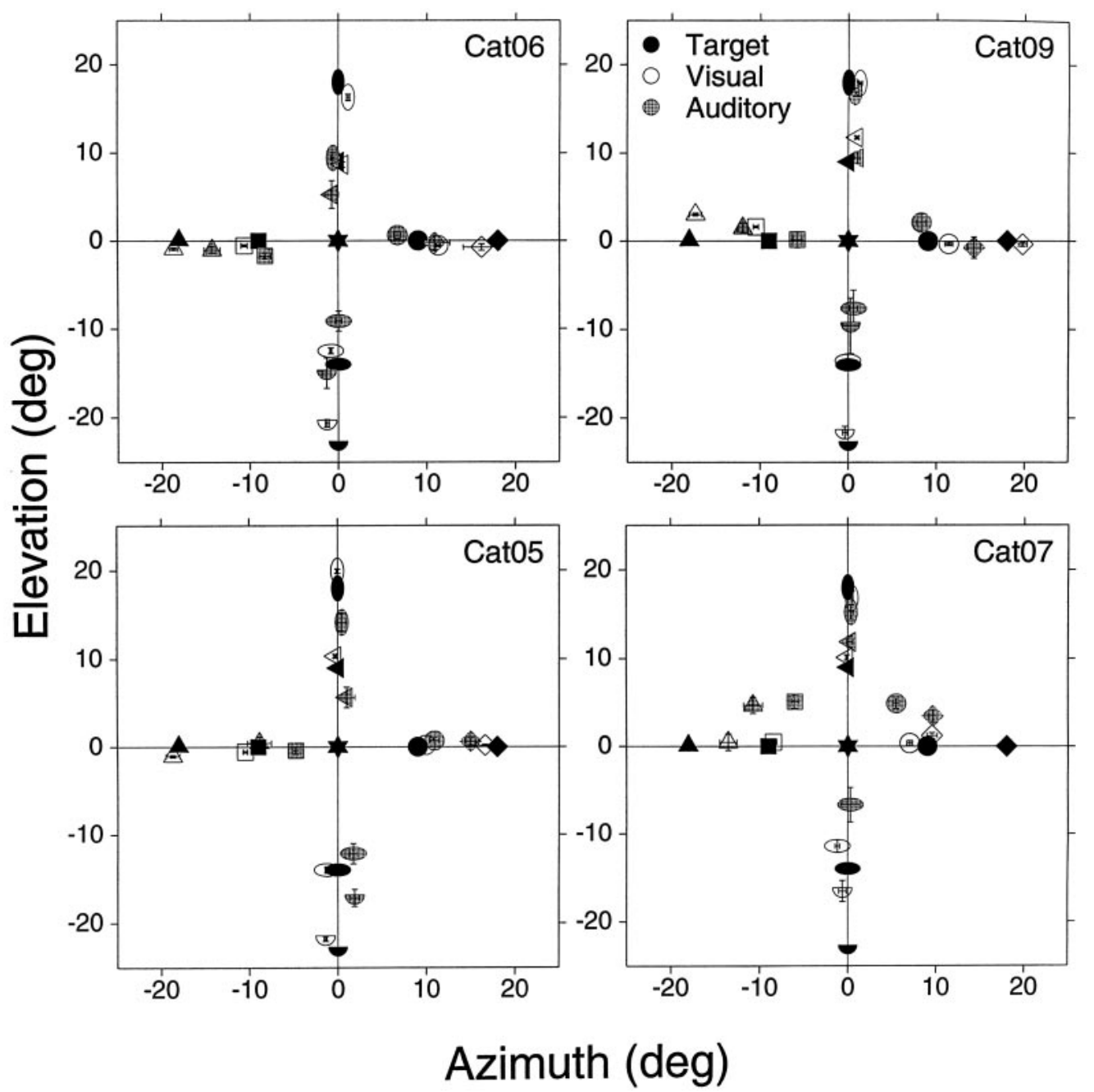

Figure 5. Mean final eye position summary for visual (open symbol) and auditory (stippled symbols) noise stimuli from four different cats with different degrees of training (Cat06, sessions 120-121; Cat09, sessions 4-7; Cat07, sessions 60-64; Cat05, sessions 72-79) to eight different targets ( filled symbols). The standard saccade task started from the primary position $\left(0^{\circ}, 0^{\circ}\right)$. Bars represent the confidence interval $(2 \times \mathrm{SE}$ of sample mean) computed for the $(x, y)$ dimensions independently. $n=1679$ trials (Cat06, 462; Cat05, 476; Cat07, 346; Cat09, 395).

stimulus was a $1500 \mathrm{msec}$ broad-band noise burst, and the visual stimulus was lit for $1500 \mathrm{msec}$.

Visual localization performance in the delayed saccade paradigm was very similar to localization in the standard saccade with slightly larger overshoots. This task was difficult for the cat to perform, as indicated by $77 \%$ no responses. Most failures were attributable to premature saccades that occurred after target onset rather than offset of the fixation LED. Overall sound localization improved slightly compared with the standard saccade task, although close inspection of the final eye position data from standard (Fig. 5, Cat06) and delayed (Fig. 9C) saccade trials revealed that the improvement was restricted to the localization of the target at $\left(18^{\circ}, 0^{\circ}\right)$, which had the largest error in the standard condition. The localization of the other three targets was not significantly different to the standard saccade.

\section{Sound localization versus remembered spatial position}

Because the visual targets were always placed at the center of a speaker, we were concerned that the cat may, after an extended period of training, saccade to locations remembered from previous visual trials rather than use acoustic cues. To test for this possibility, in one session we shifted the horizontal speakers by $4.5^{\circ}$, without presenting visual stimuli from the new locations. These test data and standard target data from a different session are shown in Figure 10. Trials involving these test targets were randomly interspersed with trials to visual and auditory targets located at other positions. The final eye position for the novel targets shifted by approximately the same amount as the targets, indicating that the cat perceived the change in location of the acoustic targets. Similar tests with novel speakers shifted from their normal positions were performed in all of our subjects with similar results.

\section{Localization of bandpass stimuli}

To examine the possible role of spectral cues in sound localization, we used narrow-bandpass noise stimuli delivered from the same speaker positions used in the broad-band localization experiments (Fig. 11). If spectral cues are important for localization, then stimuli such as these with impoverished spectral information would be expected to have the greatest effect on vertical localization, because stimuli presented from targets on the midsagittal plane do not create interaural time or level disparities.

Because only a small number of trials could be collected in each experimental session due to the low probability of presentation 

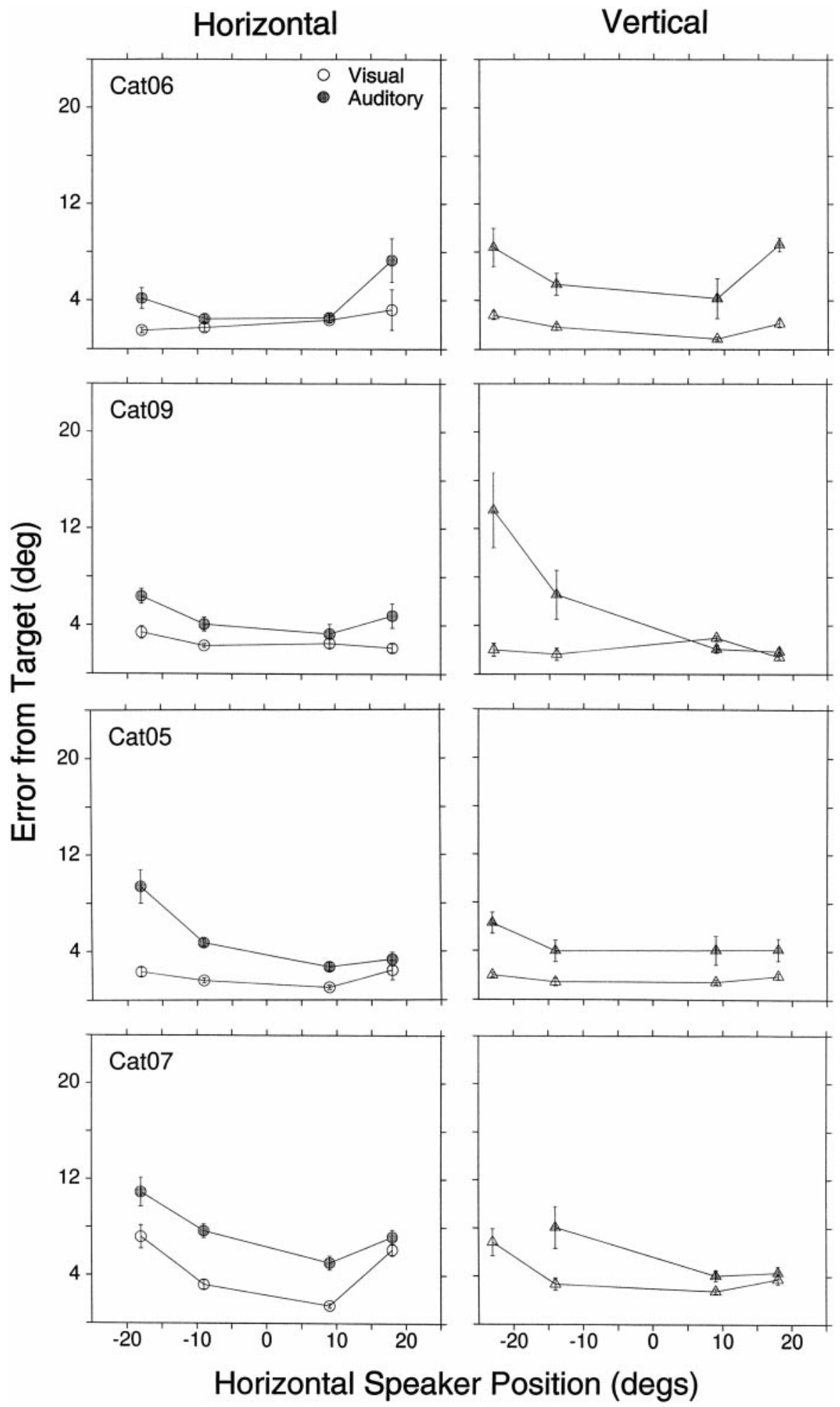

Figure 6. Mean absolute magnitude of localization errors to long-duration stimuli. Stippled symbols represent auditory (broad-band noise) data, and hollow symbols represent visual data. (see Materials and Methods), these experiments were performed on four consecutive sessions. Two subjects were tested; both localized the broad-band stimuli more accurately than any of the narrow-band stimuli. Cat06's results are shown in Figure 11; for comparison, wide-band stimulus localization is shown in the top left panel.

The results from the localization of targets on the midsagittal plane support the spectral cue hypothesis. Large localization errors of the vertical target at $\left(0^{\circ},-14^{\circ}\right)$ were observed in all five narrow-band conditions. Localization of narrow-band stimuli on the horizontal axis did not follow the expected outcome, however. We had anticipated that horizontal localization would be normal at low and high frequencies, at which interaural time and level differences would provide cues for azimuthal localization, but disrupted for stimuli in the $2-4 \mathrm{kHz}$ range. Indeed, localization was near normal at the lowest frequency and progressively disrupted as the center frequency increased, but it did not recover at the highest frequencies. For Cat09, horizontal localization was 

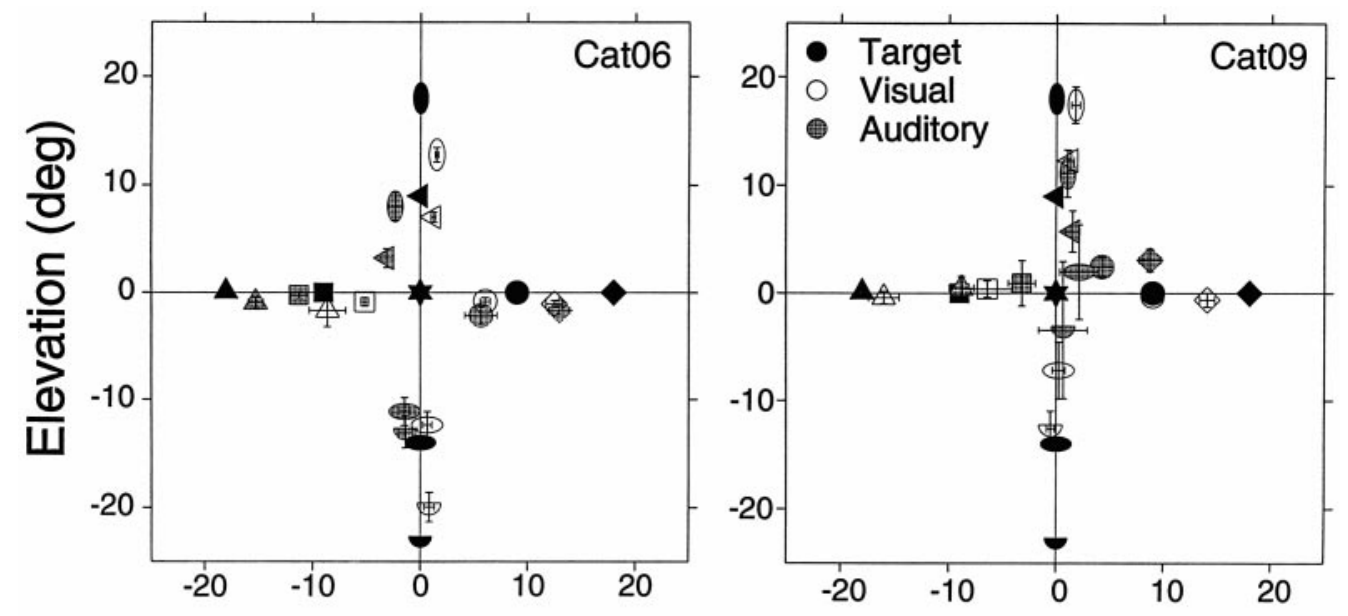

Azimuth (deg)

Figure 7. Mean final eye position summary for transient stimuli of two cats. Visual, 25 msec; auditory, single $100 \mu$ sec click. $n=1034$ (Cat06, 839 ; Cat09, 195). Details as in Figure 5.

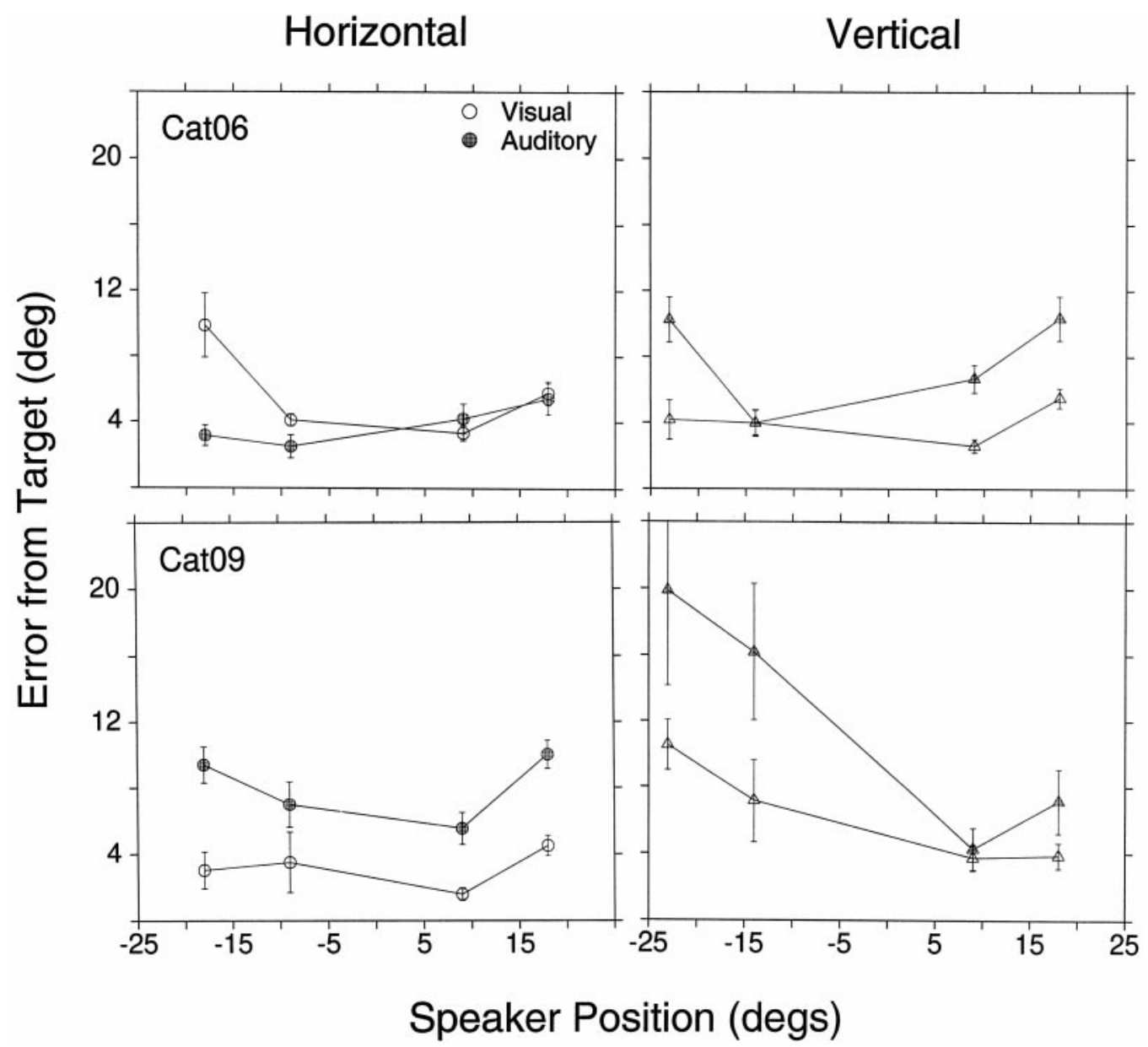

Figure 8. Mean absolute magnitude of localization errors of transient stimuli. Data from two cats (Cat06, sessions 125-137; Cat09, sessions 15-20) are shown. Details as in Figure 6.

disrupted about equally at all narrow bands used (data not shown). Interestingly, the percentage of no-response trials, which in these cases were always seen as a lack of an overt eye move- ment, was highest for the $4 \mathrm{kHz}$ bandpass condition $(1.0 \mathrm{kHz}$, $15 \% ; 2.0 \mathrm{kHz}, 26 \%$; $4.0 \mathrm{kHz}, 43 \% ; 8.0 \mathrm{kHz}, 30 \%$; and $12.0 \mathrm{kHz}$, $25 \%)$. 

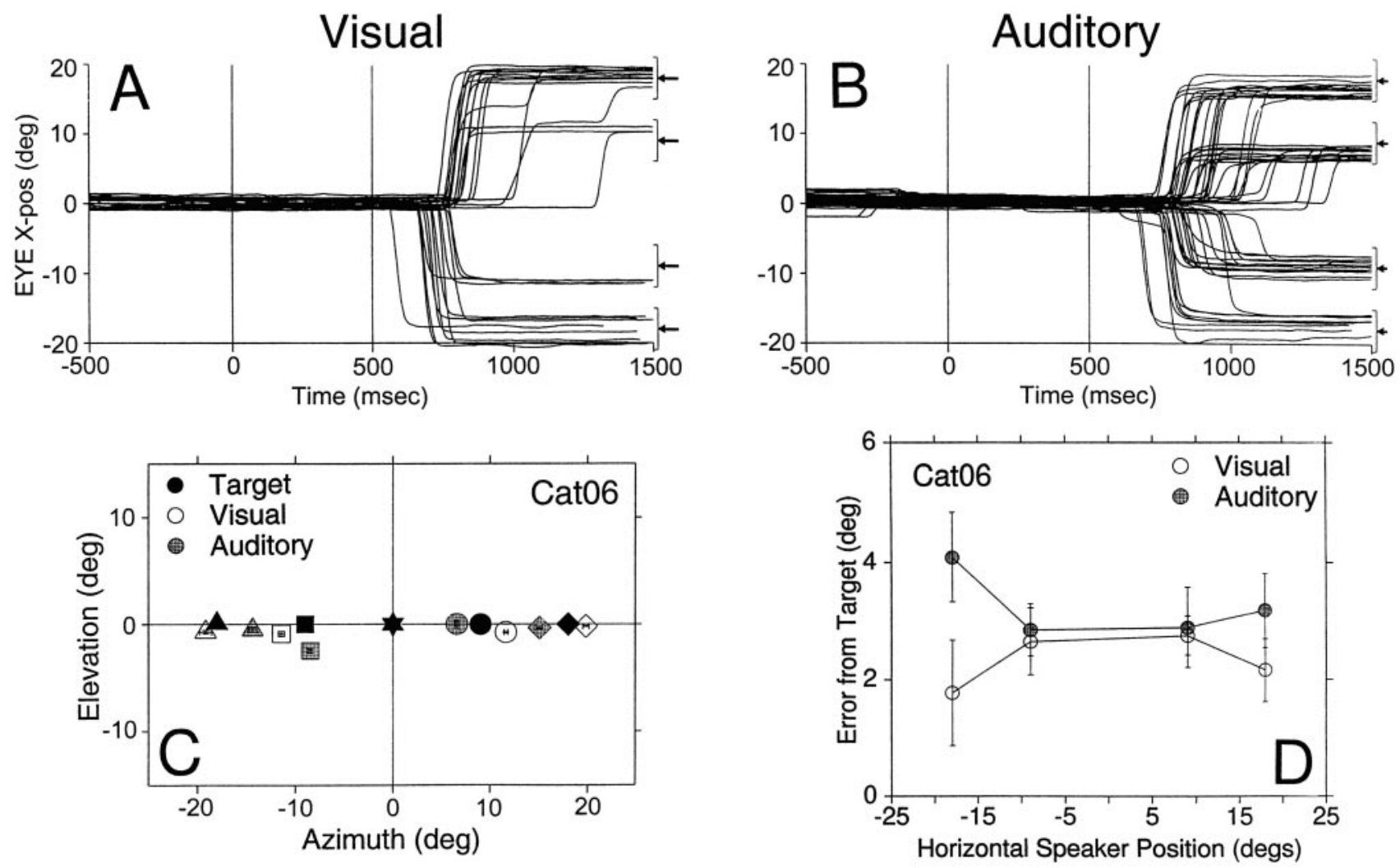

Figure 9. Sound localization with delayed saccade (Cat06; sessions 125-126). Horizontal eye position from successful trials to visual ( $A$ ) and auditory $(B)$ targets are plotted synchronized to the onset of the stimulus (thin vertical line at time $0 \mathrm{msec}$ ). The second vertical line at $500 \mathrm{msec}$ marks the offset of the fixation light. $C, D$, Mean final eye position and magnitude of localization error, respectively $(n=196)$. Details as in Figures 5 and 6 .

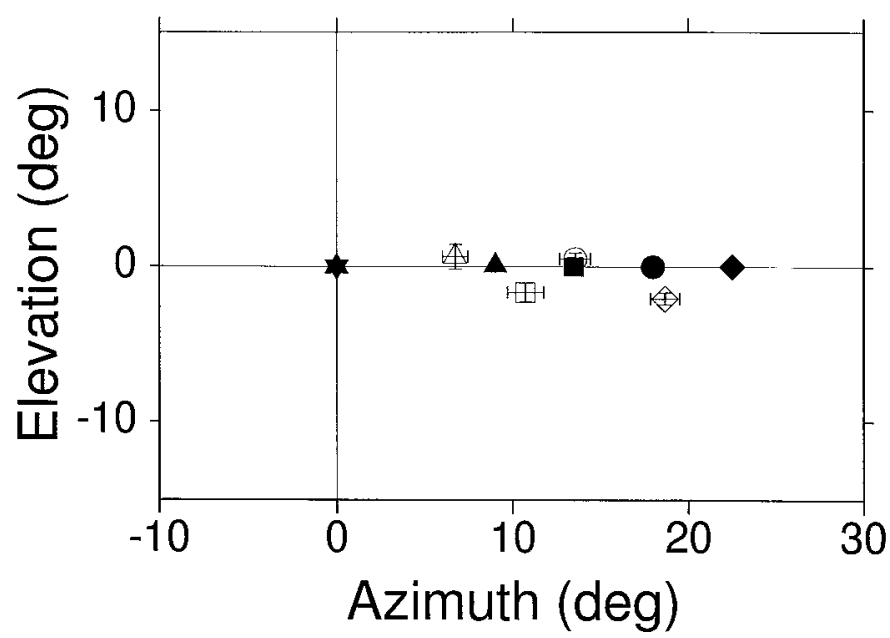

Figure 10. Sound localization control experiment. Speakers were shifted $4.5^{\circ}$ eccentrically in a single session (Cat06). The standard speaker positions are represented by the filled triangle $\left(9^{\circ}, 0^{\circ}\right)$ and circle $\left(18^{\circ}, 0^{\circ}\right)$, and the test positions are represented by the filled square $\left(13.5^{\circ}, 0^{\circ}\right)$ and diamond $\left(22.5^{\circ}, 0^{\circ}\right)$. The mean final eye position for each target is represented by the corresponding open symbols ( \pm confidence interval).

\section{Summing localization}

The results of the previous experiments demonstrate that the cats are able to look in the direction of sound sources and that their responses are based on acoustic cues. Assuming that the cats are looking to where they perceive the source of a sound, we can also study whether they experience localization illusions as do human subjects, such as the phantom sound source in a precedence effect-summing localization paradigm.

A hypothetical trial with an ICD of $>0$ is illustrated in Figure 12. To the cat the first part of a summing localization trial (Fig. $2 D$ ) was exactly like a fixation or a saccade trial that started away from the primary position. LED 1 was positioned at either $\left(-9^{\circ}, 0^{\circ}\right)$ or $\left(9^{\circ}, 0^{\circ}\right)$ so that localization of the phantom source near the midline would result in an eye movement.

Figure $13 A$ depicts the horizontal component of eye movements evoked by summing localization stimuli with various negative ICDs in Cat06. The leading speaker was to the left of the subject at $\left(-18^{\circ}, 0^{\circ}\right)$, and the lagging speaker was at $\left(18^{\circ}, 0^{\circ}\right)$. When the ICD was $0 \mu \mathrm{sec}$, the cat saccaded to positions near the midline, and as ICD duration increased, the size of the saccade toward the leading speaker also increased. The largest saccades, indicating maximal eccentricity, were evoked by standard clicks presented from the leading speaker alone (blue traces).

A summary of the results of these experiments is shown in Figure 14. Mean final eye positions, with their corresponding confidence intervals, are plotted in the $x, y$ plane for positive (Fig. $14 B$ ) and negative (Fig. 14A) ICDs. For both positive and negative ICDs, eye movement amplitude increased with ICD duration to saturate at $\sim 300 \mu \mathrm{sec}$. None of the responses to this type of stimuli was lateralized completely to the responses to the single click. Trials with single clicks from single speakers were presented from both sides and intermingled in the same series so that saccades in both directions were possible. This experiment was repeated in another cat with similar results. The responses of both animals to the $0 \mu \mathrm{sec}$ ICD exhibited a larger vertical spread compared with responses evoked by ICD $\neq 0$. 


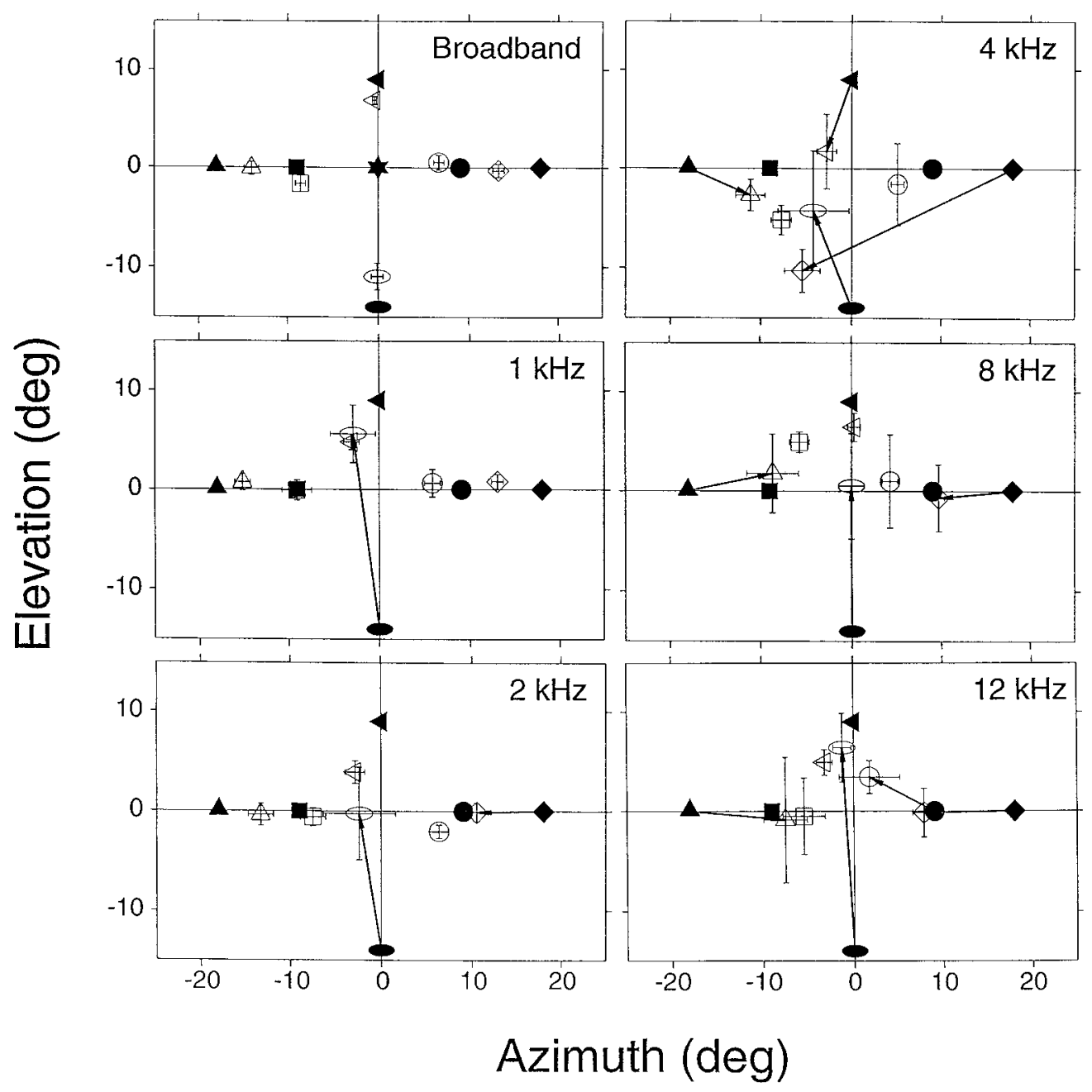

Figure 11. Localization of bandpass noise stimuli (Cat06): mean final eye position summaries for localization of broad-band and bandpass stimuli, one-sixth of an octave wide bands centered at 1,2, 4, 8, and $12 \mathrm{kHz}$. The filled symbols represent the position of the targets, and the open symbols represent the corresponding mean final eye positions. The arrows connect the targets with their corresponding final eye positions when the errors were $>7^{\circ} . n=$ 263 for bandpass stimuli. Bars represent confidence intervals.

Because human observers report that the location of the phantom source also depends on the relative level between the clicks from the two speakers (Snow, 1954), we also examined the effect of attenuating the clicks from one of the speakers, presented with 0 ICD (Fig. 13B). Three different level differences between clicks within a pair were used: $0 \mathrm{~dB}$ in turquoise, $5 \mathrm{~dB}$ in red, and $10 \mathrm{~dB}$ in blue. The data with $0 \mathrm{~dB}$ difference are the same as in Figure $14 B$. As expected, attenuating the clicks from one of the speakers resulted in a shift in the perceived location of the phantom source away from the midline in the direction of the louder click within the pair.

\section{DISCUSSION}

\section{Methodological considerations}

The results of psychophysical studies such as these can be contaminated by cues originating from the equipment that might be beyond the hearing range of the investigator but audible to the cat. To ensure that artifacts did not aid the cats, we (1) used separate amplifiers for all speakers so there was no switching transient upon speaker selection, (2) randomly roved the level of the sound from trial to trial, and (3) studied localization of target positions that were not used during training and never had asso-

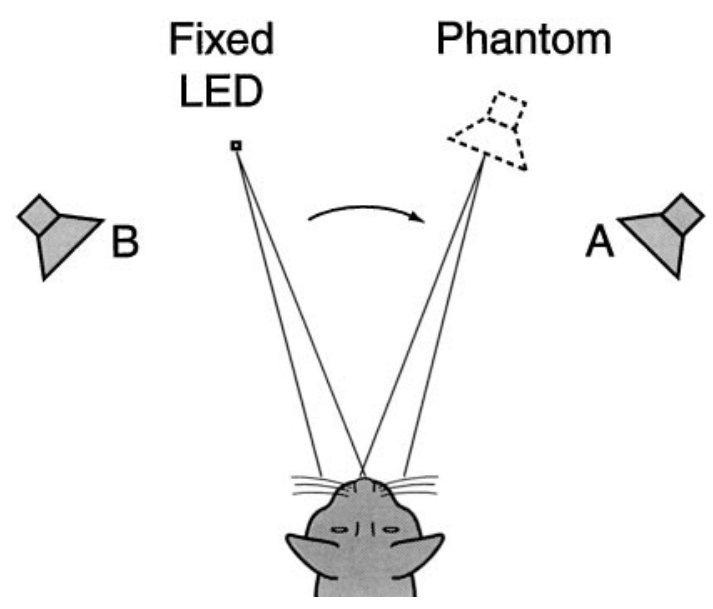

Figure 12. Schematic representation of the summing localization experiment. Pairs of clicks were presented from speakers $A$ and $B$ with an interclick delay of $<1000 \mu \mathrm{sec}$. In humans, these stimuli are perceived as a single auditory event, originating from a "phantom" source, localized to the side of the leading speaker. In this example, with the click from speaker $A$ leading the click from speaker $B$, the cat is initially required to fixate an LED to the left to facilitate an overt response. 


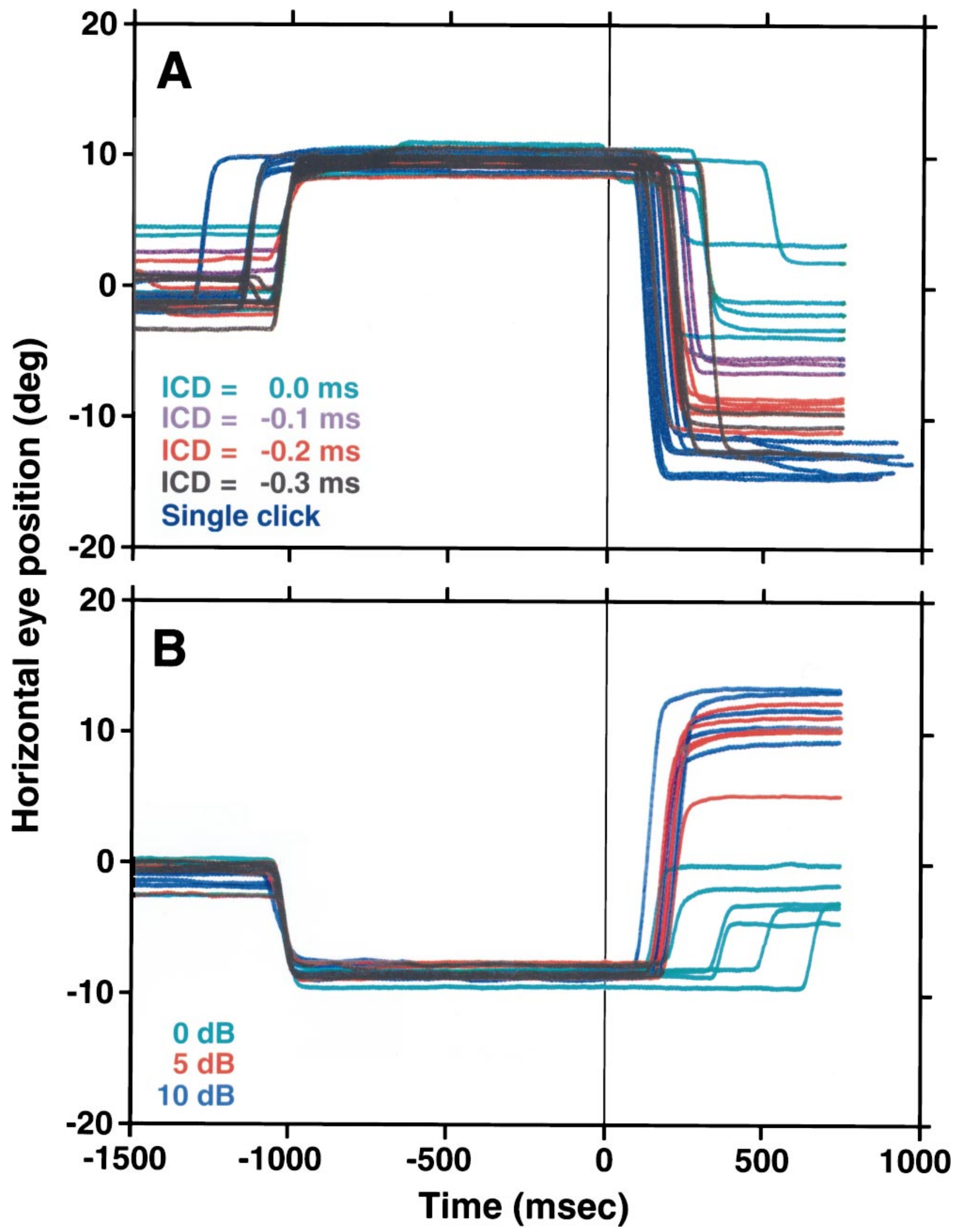

Figure 13. Summing localization. Horizontal eye position traces are plotted synchronized to the onset of the acoustic stimuli at time 0 msec. $A$, ICD series: turquoise, $0 \mu \mathrm{sec}$; purple, $-100 \mu \mathrm{sec}$; red, $-200 \mu \mathrm{sec}$; black, $-300 \mu \mathrm{sec} ;$ blue, single clicks. The cat was required to fixate an LED at $\left(9^{\circ}, 0^{\circ}\right)$ for $1000 \mathrm{msec}$ and then was expected to saccade where it perceived sound to originate. $B$, Intensity series. The independent variable was the attenuation of the clicks presented from the speaker at $\left(-18^{\circ}, 0^{\circ}\right)$ : turquoise, $0 \mathrm{~dB} ;$ red, $5 \mathrm{~dB} ;$ blue, $10 \mathrm{~dB}$; thus the cat was required to initially fixate an LED at $\left(-9^{\circ}, 0^{\circ}\right)$. With nonzero attenuations the phantom sources were expected to be perceived to the right of the midline. Data from the mirror image experiments are not shown. 


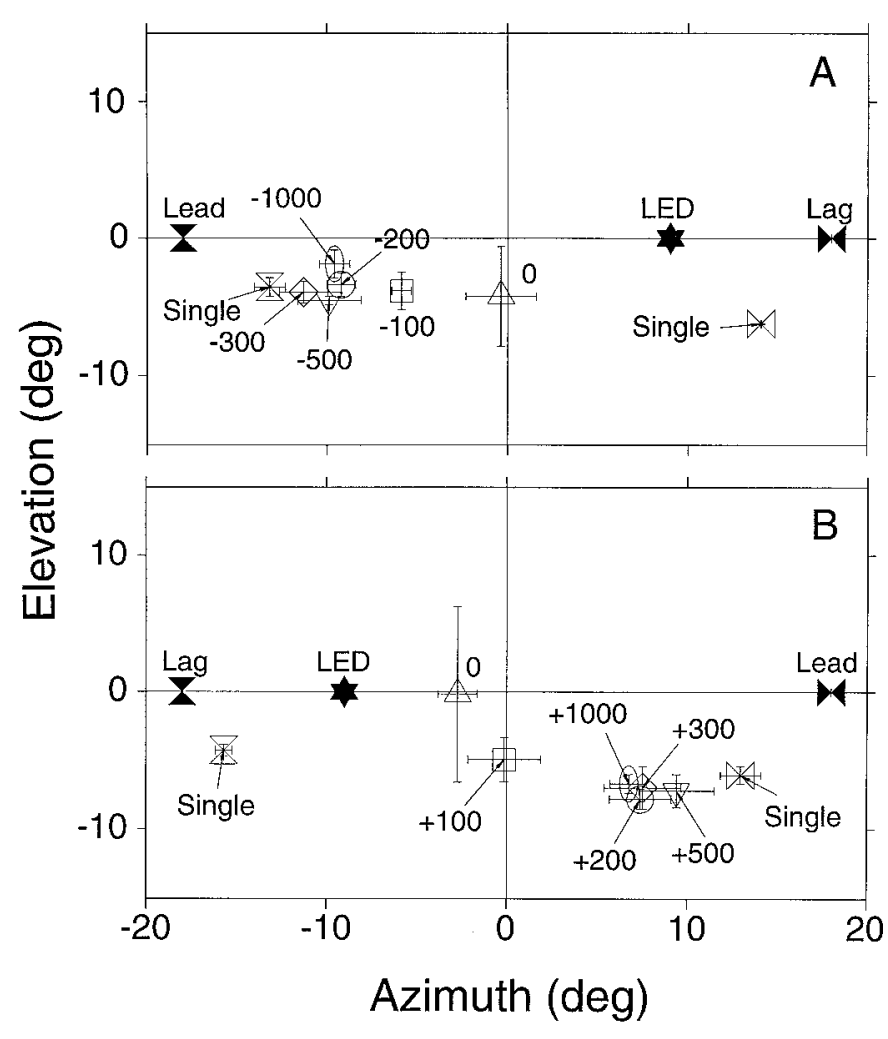

Figure 14. Summing localization: ICD series summary. Mean final eye position from the negative $(A ; n=36$ trials) and the positive $(B ; n=42$ trials) ICD conditions. The position of the fixation LED is illustrated by a filled star; the position of the leading (Lead) and lagging (Lag) speakers are labeled. The mean final eye positions for the various ICDs are illustrated by the open symbols. Bars represent the confidence intervals computed as in Figure 5. The point for the single click presented from the right speaker in $A$ represents two data points only.

ciated visual targets (Fig. 10). In addition, we never presented blocks of identical trials, which would add predictability as a confounding variable. We are, therefore, confident that the present results are uncontaminated by artifacts.

The combined visual-auditory stimuli used during the first stages of training and the randomly intermixed visual and auditory trials presented from the same locations during testing sessions raise the possibility that in auditory trials cats might have used cues derived from visual trials. Several lines of evidence suggest that our cats were localizing sound and not responding from memory. First, when auditory stimuli were presented from novel locations that were never associated with visual targets, the cats appropriately shifted their final eye position (Fig. 10). Second, because all of the behavioral experiments and daily training sessions involved many different combinations of visual and auditory targets and tasks from many positions, and all were delivered randomly, the sheer number of different combinations makes it unlikely that memory or learning played any role. Third, Figure 5 shows that final eye position for both visual and auditory conditions were different for most targets, although LEDs and speakers were located in the same spatial locations. If the cats had responded from memory, final eye position across modalities should overlap. Finally, the speakers were hidden from view so the cat could not have associated sounds to any visual cue. Taken together, these results suggest that our cats used acoustic information to look to auditory targets and not cues learned from visual stimuli.
In some cats we observed biases toward or against localizing sound in particular areas of the field; e.g., some cats were reluctant to saccade to targets to the left, and others were reluctant to saccade to targets to the right. Because these biases would sometimes change over the weeks and months of training, damage to the oculomotor plant when inserting the fine-wire coils underneath the conjunctiva or differences in speaker characteristics cannot account for these biases.

\section{Localization of broad-band stimuli}

The paradigm developed for this series of studies required the cat to make eye movements to the sources of auditory and visual stimuli. Spatial errors could be attributable to mislocalization of the targets or failure of the oculomotor system to perform the correct eye movement. The high accuracy of saccades to visual, compared with acoustic, targets (Fig. 5) makes us attribute the major component of errors in auditory trials to inaccurate sound localization.

With some exceptions, our results from the head-fixed cat are consistent with those from the head-free cat (Beitel and Kaas, 1993; May and Huang, 1996), monkey, and human performing similar tasks (Zahn et al., 1978, 1979; Zambarbieri et al., 1982; Jay and Sparks, 1990; Frens and Van Opstal, 1995), but inferior in accuracy to those of the owl (Knudsen et al., 1979).

Our cats consistently undershot auditory targets, with errors that increased in magnitude with target eccentricity (Figs. 5, 6, 10). Beitel and Kaas (1993) and May and Huang (1996) reported similar increases in localization error with target eccentricity in the unconditioned and conditioned, respectively, head-free cat, although direct comparisons are difficult because of different experimental conditions. Whereas we measured eye movements with the head fixed, Beitel and Kaas (1993) and May and Huang (1996) measured head movements without regard to eye position. In addition, they tested stimuli in more eccentric positions; May and Huang (1996) delivered two bursts to facilitate corrective head movements, and Beitel and Kaas (1993) used blind cats. Nevertheless, the results are remarkably similar.

May and Huang (1996) suggested that undershooting results from poorly defined head-related transfer functions (HRTFs) (Musicant et al., 1990) for source positions beyond $\pm 25^{\circ}$ azimuth, because their cats were able to reduce a large initial error when presented with a second noise burst after the initial response was completed. The head of the cat was presumably within $\pm 25^{\circ}$ of the target at the time of the second stimulus presentation. Our observations were obtained over a smaller azimuthal range (within $20^{\circ}$ from the midsagittal plane) and, therefore, must be attributable to other factors.

This increased undershooting of auditory targets with target eccentricity appears to be a common feature of localization behavior, for it has been observed in other species, such as the barn owl (Knudsen et al., 1979), under different experimental conditions. Interestingly, the owl does not reduce the magnitude of its errors for the most peripheral targets to a level comparable to the most central (less than $\pm 30^{\circ}$ ) targets while localizing long stimuli durations, which provide feedback as the animal executes its response (Knudsen et al. 1979).

The data of Jay and Sparks (1990) of broad-band localization from the monkey showed a similar effect, but surprisingly, their human data, which were collected under similar experimental conditions, did not. These results of Jay and Sparks (1990) from human subjects differ from those of Zahn et al. (1978), which showed an increase in the magnitude of the error with target 
eccentricity. Such differences may be attributable to the use by Zahn et al. (1978) of narrow-band noise instead of broad-band stimuli.

Target duration affected localization in our cats in both the visual and auditory modalities. The magnitude of sound localization errors increased considerably when transient stimuli were used in the two cats tested, particularly for targets below the horizontal plane. There was also an overall reduction in accuracy for visual targets.

The size of the error in the single-click condition was approximately double compared with the long-duration stimuli condition. There is evidence that performance in some localizationrelated tasks improves with stimulus duration (Yost et al., 1971; Nuetzel and Hafter, 1976; see Heffner and Heffner, 1988). Beitel and Kaas (1993) reported that horizontal localization accuracy increased with stimulus duration. For the longest target duration $(1500 \mathrm{msec})$ presented in the frontal hemifield, their cats virtually canceled the errors made in the primary response with corrective responses. May and Huang (1996), on the other hand, did not see an increase in accuracy as a function of duration, but their longest stimulus was only $200 \mathrm{msec}$. Their cats canceled the errors made in the initial response to a $40 \mathrm{msec}$ noise burst, after a second burst was presented. Finally, it appears that the ability of cats to localize transient stimuli is inferior to that of the owl (Knudsen et al., 1979), in which localization performance is essentially identical for short- $(75 \mathrm{msec})$ and long-duration broadband stimuli within the frontal $\pm 30^{\circ}$.

\section{Localization of narrow-band stimuli}

Overall localization accuracy of narrow-band stimuli was affected compared with broad-band stimuli. As predicted by the spectral cue hypothesis (Middlebrooks and Green, 1990), localization of vertical targets was most affected. The cat's perceptions of the target at $\left(0^{\circ},-14^{\circ}\right)$ were severely altered at all narrow-band frequencies tested. For targets located along the horizontal axis, localization accuracy was also affected, but not in a manner totally consistent with our predictions, because performance was also poor at higher frequencies ( 8 and $12 \mathrm{kHz}$ bands), despite robust interaural level cues. We infer that the higher percentage of no-response trials at $4.0 \mathrm{kHz}$ reflects more difficulty in localizing the sound.

Although consistent with the spectral cue hypothesis, our results do not fully replicate those from human subjects, who mislocalized the vertical component of narrow-band noise bursts to areas of space where HRTFs have a prominent peak at the center frequency of the noise, regardless of the position of the source (Middlebrooks, 1992). Huang and May (1996) also found systematic mislocalizations along the vertical plane in the headfree cat, but they favor an explanation based on the shifting position of the first notch in HRTFs of vertical sources.

The differences between the present results and those of Middlebrooks (1992) and Huang and May (1996) could be attributable to our head-fixed preparation and the limited oculomotor range of the cat. Possibly, the cats perceived the sound as originating from areas beyond their oculomotor range, which caused an inappropriate response or simply a wait for the next trial.

\section{Summing localization}

The data presented in Figures 13 and 14 constitute the first direct demonstration that cats experience summing localization. In addition, they provide information on the temporal limits of this illusion in cats. For $0 \mu \mathrm{sec}$ ICDs, the cat directed its eyes to a position near the center of the field, starting from either the left or the right fixation LEDs. As the ICDs were increased the final eye position was more eccentric, always to the side of the leading click, saturating at $\sim 300 \mu \mathrm{sec}$. In a human, whose head is larger than that of a cat, the saturating ICD is $\sim 800-1000 \mu \mathrm{sec}$ (Blauert, 1983). With some variability the effects were reproduced on both sides (Fig. 14A,B).

It is important to point out that this is a difficult task to perform. To the human listener at least, the clicks originating from the phantom source sound different from single clicks (Blauert, 1983; Perrott et al., 1987, 1989; Litovsky and Macmillan, 1994). This is an aspect of the task that some cats may find confusing.

\section{REFERENCES}

Beitel RE, Kaas JH (1993) Effects of bilateral and unilateral ablation of auditory cortex in cats on the unconditioned head orienting response to acoustic stimuli. J Neurophysiol 70:351-369.

Blauert J (1983) Spatial hearing: the psychophysics of human sound localization. Cambridge, MA: MIT.

Butler RA (1975) The influence of the external and middle ear on auditory discriminations. In: Handbook of sensory physiology, V, Auditory System, Pt 2 (Keidel WD, Neff WD, eds), pp 247-260. Berlin: Springer.

Casseday JH, Neff WD (1973) Localization of pure tones. J Acoust Soc Am 54:365-372.

Cranford JL (1982) Localization of paired sound sources in cats: effects of variable arrival times. J Acoust Soc Am 72:1309-1311.

Frens MA, Van Opstal AJ (1995) A quantitative study of auditoryevoked saccadic eye movements in two dimensions. Exp Brain Res 107:103-117.

Fuchs AF, Robinson DA (1966) A method for measuring horizontal and vertical eye movements chronically in the monkey. J Appl Physiol 21:1068-1070.

Gardner MB, Gardner RS (1973) Problem of localization in the median plane: effect of pinnae cavity occlusion. J Acoust Soc Am 53:560-575.

Haas H (1951) The influence of a single echo on the audibility of speech. Acustica 1:49-58.

Hartmann WM (1983) Localization of sound in rooms. J Acoust Soc Am 74:1380-1391.

Hebrank J, Wright D (1974a) Are two ears necessary for the localization of sound sources on the median plane? J Acoust Soc Am 56:935-938.

Hebrank J, Wright D (1974b) Spectral cues used in the localization of sound sources on the median plane. J Acoust Soc Am 56:1829-1834.

Heffner RS, Heffner HE (1988) Sound localization acuity in the cat: effect of azimuth, signal duration, and test procedure. Hear Res 36:221-232.

Huang AY, May BJ (1996) Sound orientation behavior in cats. II. Midfrequency spectral cues for sound localization. J Acoust Soc Am 100:1070-1080.

Irvine DRF (1986) The auditory brainstem: a review of the structure and function of auditory brainstem processing mechanisms. Berlin: Springer.

Jay MF, Sparks DL (1990) Localization of auditory and visual targets for the initiation of saccadic eye movements. In: Comparative perception, basic mechanisms, III (Berkley MA, Stebbins WC, eds), pp 351-374. New York: Wiley.

Jenkins WM, Masterton RB (1982) Sound localization: effects of unilateral lesions in central auditory system. J Neurophysiol 47:987-1016.

Jenkins WM, Merzenich MM (1984) Role of cat primary auditory cortex for sound-localization behavior. J Neurophysiol 52:819-847.

Judge SJ, Richmond BJ, Chu FC (1980) Implantation of magnetic search coils for measurement of eye position: an improved method. Vision Res 20:535-538.

Knudsen EI, Blasdel GG, Konishi M (1979) Sound localization by the barn owl (Tyto alba) measured with the search coil technique. J Comp Physiol 133:1-11.

Litovsky RY, Macmillan NA (1994) Sound localization precision under conditions of the precedence effect: effects of azimuth and standard stimuli. J Acoust Soc Am 96:752-758.

Martin RL, Webster WR (1987) The auditory spatial acuity of the do- 
mestic cat in the interaural horizontal and median vertical planes. Hear Res 30:239-352.

May BJ, Huang AY (1996) Sound localization behavior in cats. I. Localization of broadband noise. J Acoust Soc Am 100:1059-1069.

May BJ, Aleszcyk CM, Sachs MB (1991) Single-unit recording in the ventral cochlear nucleus of behaving cats. J Neurosci Methods 40:155-169.

McHaffie JG, Stein BE (1983) A chronic headholder minimizing facial obstructions. Brain Res Bull 10:859-860.

Middlebrooks JC (1992) Narrow-band sound localization related to external ear acoustics. J Acoust Soc Am 92:2607-2624.

Middlebrooks JC, Green DM (1990) Directional dependence of interaural envelope delays. J Acoust Soc Am 87:2149-2162.

Mills (1958) On the minimum audible angle. J Acoust Soc Am 30:237-246.

Musicant AD, Chan JCK, Hind JE (1990) Direction-dependent spectral properties of cat external ear: new data and cross-species comparisons. J Acoust Soc Am 87:757-781.

Nuetzel JM, Hafter ER (1976) Lateralization of complex waveforms: effects of fine structure, amplitude, and duration. J Acoust Soc Am 60:1339-1346.

Perrott DR, Strybel TZ, Manligas CL (1987) Conditions under which the Haas precedence effect may or may not occur. J Aud Res 27:59-72.

Perrott DR, Marlborough K, Merrill P, Strybel TZ (1989) Minimum audible angle and thresholds obtained under conditions in which precedence effect is assumed to operate. $\mathrm{J}$ Acoust Soc Am 85:282-288.

Phillips DP, Brugge JF (1985) Progress in neurophysiology of sound localization. Annu Rev Psychol 36:245-274.

Phillips DP, Calford MB, Pettigrew JD, Aitkin LM, Semple MN (1982) Directionality of sound pressure transformation at the cat's pinna. Hear Res 8:13-28.

Populin LC, Yin TCT (1995) Behavioral studies of sound localization in the cat. Soc Neurosci Abstr 21:129.

Populin LC, Yin TCT (1996) Behavioral studies of the precedence effect: the summing localization period. Assoc Res Otolaryngol Abstr 753.
Rhode WS (1976) A digital system for auditory neurophysiological research. In: Current computer technology in neurobiology (Brown P, ed), pp 543-567. Washington DC: Hemisphere.

Rice JJ, May BJ, Spirou GA, Young ED (1992) Pinna-based spectral cues for sound localization in cat. Hear Res 58:132-152.

Snow WB (1954) Effect of arrival time on stereophonic localization. J Acoust Soc Am 26:1071-1074.

Stevens SS, Newman EB (1936) Localization of actual sources of sound. Am J Psychol 48:297-306.

Thompson GC, Masterton RB (1978) Brain stem auditory pathways involved in reflexive head orienting to sound. J Neurophysiol 41:1183-1202.

Wallach H, Newman EB, Rosenzweig MR (1949) The precedence effect in sound localization. Am J Psychol 57:315-336.

Wiener FM, Pfeiffer RR, Backus SN (1966) On the sound pressure transformation by the head and auditory meatus of the cat. Acta Otolaryngol 61:255-269.

Wightman FL, Kistler DJ (1994) Sound localization. In: Human psychophysics (Yost WA, Popper AN, Fay RR, eds), pp. 155-192. Berlin: Springer.

Yin TCT (1994) Physiological correlates of the precedence effect and summing localization in the inferior colliculus of the cat. J Neurosci 14:5170-5186.

Yin TCT, Joris PX, Smith PH, Chan JCK (1997) Neural mechanisms underlying the coding of interaural time differences. In: Binaural and spatial hearing in real and virtual environments (Gilkey R, Anderson T, eds), pp 427-445. New York: Erlbaum.

Yost WA, Wightman FL, Green DM (1971) Lateralization of filtered clicks. J Acoust Soc Am 50:605-611.

Zahn JR, Abel LA, Dell'Osso LF (1978) Audio-ocular response characteristics. Sens Processes 2:32-37.

Zahn JR, Abel LA, Dell'Osso LF, Daroff RB (1979) The audioocular response: intersensory delay. Sens Processes 3:60-65.

Zambarbieri D, Schmid R, Magenes G, Prablanc C (1982) Saccadic responses evoked by presentation of visual and auditory targets. Exp Brain Res 47:417-427. 